\title{
« Retrouver les choses par le dedans »
}

La « recréation organique » au principe des Hommes de bonne volonté de Jules Romains

\section{Sophie Guermès}

\section{(2) OpenEdition}

1 Journals

Édition électronique

URL : https://journals.openedition.org/genesis/1642

DOI : $10.4000 /$ genesis. 1642

ISSN : 2268-1590

Éditeur :

Presses universitaires de Paris Sorbonne (PUPS), Société internationale de génétique artistique littéraire et scientifique (SIGALES)

Édition imprimée

Date de publication : 9 mai 2016

Pagination : 97-105

ISBN : 9791023105315

ISSN : $1167-5101$

\section{Référence électronique}

Sophie Guermès, « «Retrouver les choses par le dedans » », Genesis [En ligne], 42 | 2016, mis en ligne le 05 juin 2017, consulté le 17 janvier 2023. URL : http://journals.openedition.org/genesis/1642 ; DOI : https://doi.org/10.4000/genesis.1642 


\section{«Retrouver les choses par le dedans» La "recréation organique» au principe des Hommes de bonne volonté de Jules Romains}

Sophie Guermès

\section{«Système Balzac - Système Zola »}

peut-on lire dans les notes préparant une conférence sur le roman, après la publication des tomes VII et VIII des Hommes de bonne volonté 1 . Jules Romains refuse les systèmes, auxquels il oppose un «travail organique ${ }^{2}$ ». Dans la préface de 1932, il reproche implicitement à l'auteur de La Comédie humaine, explicitement à celui des RougonMacquart une unité de façade, et dénie même à ce dernier d'avoir retracé l'histoire «concrète» d'une famille, l'hérédité devenant à ses yeux un principe aussi artificiel qu'abstrait ${ }^{3}$. Jules Romains va éviter le plus possible les pièges du sériel par la traduction littéraire de l'unanimisme et de la simultanéité; quant au thème familial, il ne sera pas absent de ses volumes, mais à la condition de ne pas en faire une idée générale. Il pense avoir trouvé l'antidote dans la période qu'il retrace :

dynamisme aussi peu artificiel que possible

celui de la coulée de civilisation

Époque particulièrement émouvante $[\ldots]$

Sujet véritable :

La révolution que nous sommes en train de vivre ${ }^{4}$

La «coulée» implique la fluidité; l'image de la révolution appelle celle du cycle. Le cycle permet de rompre avec «une action rectiligne» aussi bien qu'avec tout ce qui pourrait donner l'impression d'une mécanique. Empruntant beaucoup à Zola (méthodes de travail, documentation ${ }^{5}$, connaissance des milieux...; succession de romans individuels regroupés sous un titre général) et ne rompant pas avec le récit à la troisième personne, Jules Romains peut surtout espérer innover sur le plan macrostructural. Son «roman de dimensions inusitées ${ }^{6}$ » sera donc motivé par la volonté d'opposer aux Rougon-Macquart, qu'il juge une construction factice et simplificatrice, le «mouvement naturel ${ }^{7} \gg$ d'une élaboration rendant compte du foisonnement du réel. Jules Romains retourne le naturalisme contre lui-même en opposant constamment le «naturel» qu'il souhaite restituer dans son cycle à l' «artifice» qu'il dénonce chez Zola. À la «routine», il répondra par la rupture, à la linéarité, par l'imprévu et les

1. Cahiers Jules Romains 5, «Les dossiers préparatoires des Hommes de bonne volonté, le projet initial et l'élaboration des quatre premiers volumes (1923-1932). Textes de Jules Romains», présenté par Annie Angremy, Paris, Flammarion, 1982, p. 244. Ces notes sont de la fin de l'année 1934 ou du début de l'année 1935.

2. Ibid. : «avant tout travail organique - retrouver les choses par le dedans ». Dans la préface des Hommes de bonne volonté, il écrit : «Car, j'y insiste, il n'est pas question de remplacer un artifice par un autre, une routine par une autre, et de faire de ce mode de composition un usage qui procèderait de l'esprit de système, et nous éloignerait à nouveau d'une réalité inépuisable en cheminements et en ressources » (Paris, Robert Laffont, coll. «Bouquins», 1988, p. 8).

3. De fait, le plan «unanimiste» (1932) des Hommes de bonne volonté, reproduit ici (fig. 1), est très différent de ceux des Rougon-Macquart (nous reproduisons le dernier, datant de 1893 , fig. 2). Non plus un tronc et des ramifications, mais des sortes de monades, traduisant, selon la lecture que fit Husserl des monades dans ses Méditations cartésiennes, à la fois la conscience individuelle et l'intersubjectivité. La coïncidence est frappante. Peut-être Jules Romains, agrégé de philosophie, avait-il assisté aux conférences données par Husserl à la Sorbonne en février 1929, et qui furent traduites (par Emmanuel Levinas et Gabrielle Pfeiffer) puis publiées en France en 1931. Il n'en a pas moins considéré l'unanimisme comme un «anti-monadisme» (Problèmes d'aujourd'hui, Paris, Kra, 1931, p. 165). 4. Ibid., p. 243.

5. Voir Cahiers Jules Romains 6, «Les dossiers préparatoires des Hommes de bonne volonté, les tomes V à XIV et les tomes XVII à XXVII (19321944). Textes de Jules Romains », présenté par Annie Angremy, Paris, Flammarion, 1985, p. 93-98. L'ouvrage présente les tomes V à XIV et les tomes XVII à XXVII (1932-1944) des dossiers préparatoires. La documentation est d'autant plus importante que le romancier ne connaît pas bien ce dont il compte parler. Le Vatican en est l'exemple le plus probant. S'y ajoutent la Première Guerre mondiale (même s'il l'a vécue, il a besoin de s'appuyer sur des archives pour tout reconstituer), et les débuts de l'URSS. 6. Jules Romains, Les Hommes de bonne volonté, op. cit., 1988, t. I, préface (février 1932), p. 2.

7. Ibid., p. 8. 


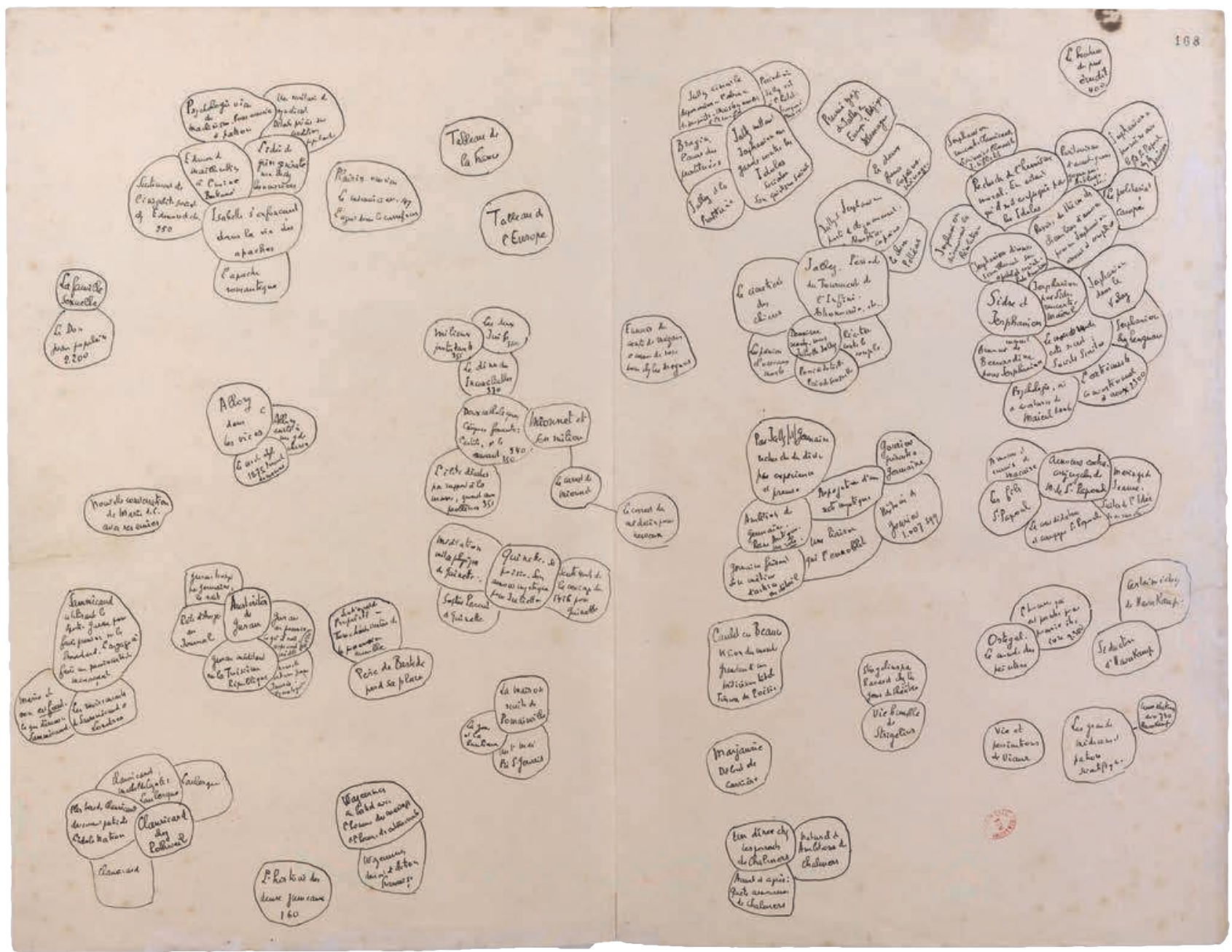

Fig. 1 : Jules Romains, Plan «unanimiste» (1932) des Hommes de bonne volonté (BnF, Fonds Jules Romains, t. I, fos 167 vo-168 ro)

«efforts zigzagants », au déterminisme, par l'incertitude et le «pullulement non orienté 8 ». Et surtout, à l'extériorité, par l'intériorité d'une composition conçue comme un tout organique. C'est pour cette raison qu'il rejette à la fois le terme de «série» et celui de « cycle $^{9}$ » pour définir ce qu'il élabore, et qu'il conçoit comme un seul très long roman. C'est qu'entre Zola et Jules Romains, il y a eu Proust. Bien que non cité, parce que n'ayant pas influencé directement Jules Romains, l'auteur d'À la recherche du temps perdu a enseigné aux happy few qui l'avaient lu une autre façon de concevoir une totalité romanesque. L'examen des dossiers des Hommes de bonne volonté, et de la construction des différents volumes, permettra de mesurer dans quelle mesure Jules Romains a réussi à s'affranchir du «système Zola».

La préface aux Hommes de bonne volonté, publiée en février 1932 en accompagnement des deux premiers volumes de sa série, pourrait être sous-titrée «Différences entre Zola et moi », sur le modèle du feuillet où Zola, encore quasiment inconnu, mais concevant Les Rougon-Macquart en pleine connaissance de ses moyens créateurs, se mesurait à l'auteur de La Comédie humaine ${ }^{10}$. Jules Romains prône «le mouvement et la multiplicité» dans sa «vaste fiction en prose » visant à rendre compte du «monde moderne». Il présente ses livres précédents comme des essais préparant la traduction littéraire sur une grande échelle de la prise de conscience, en 190811, de l'unité de tous les éléments apparemment disparates qui composent le monde. Cette sorte d'illumination, il l'a intitulée « unanimisme», et en

8. Ibid., p. 9.

9. Ibid., p. 2

10. «Différences entre Balzac et moi», BnF, NAF 10345, fos 14-15.

11. C'est la raison pour laquelle il ne reste aucune trace écrite du projet initial. Celui-ci s'est constitué lentement, au fil des années, et n'a abouti que progressivement à une traduction romanesque. 


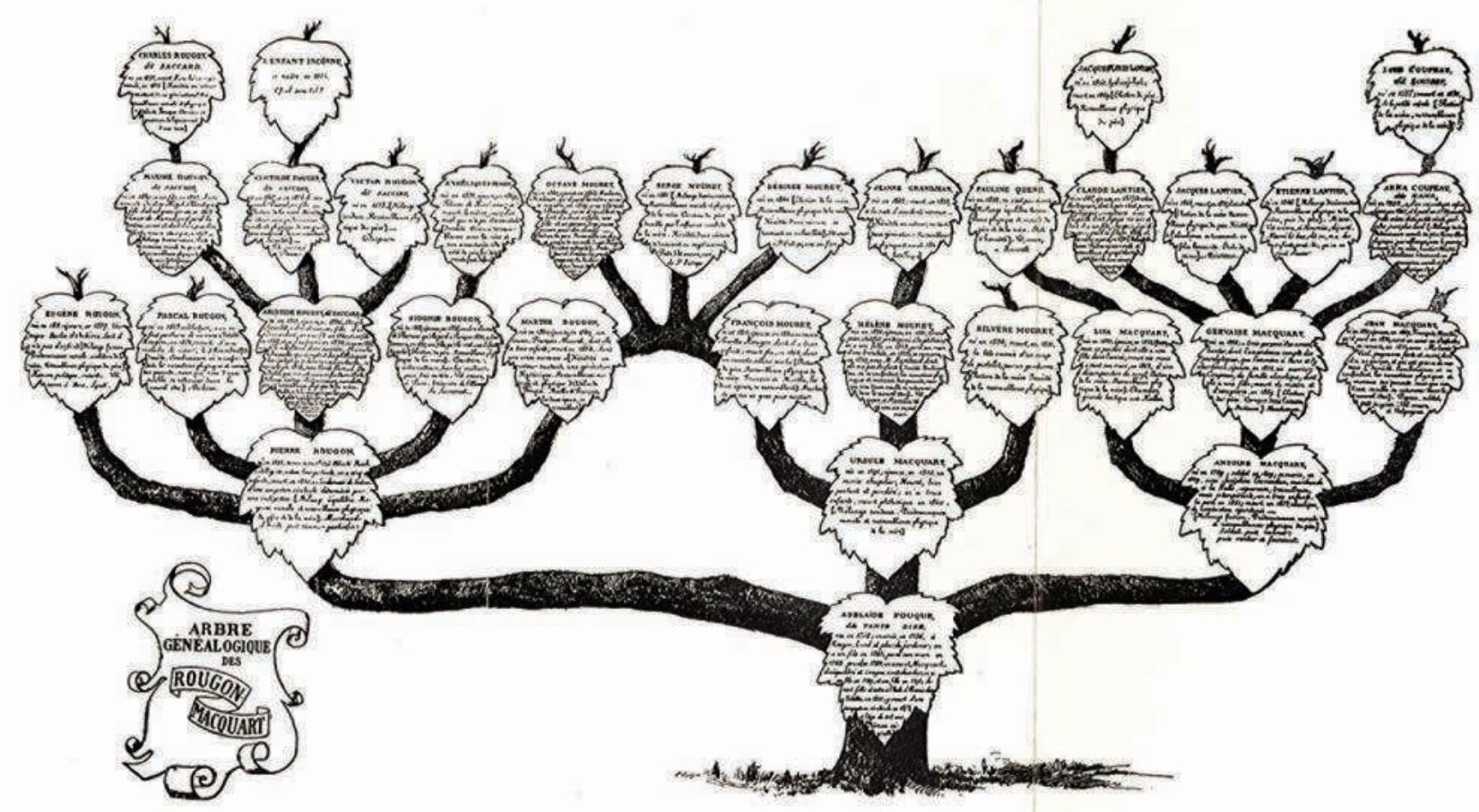

Fig. 2 : Émile Zola, Arbre généalogique des Rougon-Macquart (1893)

avait donné très tôt la traduction poétique dans le recueil La Vie unanime. Le dynamisme au principe même de la série, l'écriture de la simultanéité, l'éloignent d'emblée de Balzac comme de Zola, qui avaient choisi comme soubassements respectifs de La Comédie humaine et des Rougon-Macquart des notions stables (en dépit des accidents de l'Histoire), la monarchie et la religion, ou une vérité scientifique peu suspecte d'être niée (en dépit des excès déterministes de Zola), l'hérédité. Il y avait dans ces points d'ancrage initiaux une immobilité 12 qui ne convient pas à la vision du monde que Jules Romains veut transmettre dans Les Hommes de bonne volonté. Il n'en reste pas moins vrai que ces cycles et ces séries dont l'unité a été mûrie avant l'écriture des romans (Zola, Jules Romains), ou s'est imposée en cours d'élaboration (Balzac), ont pour point commun un principe extérieur au domaine strictement littéraire : les romans sont conçus comme des démonstrations, même s'ils évitent dans leur facture le didactisme pesant - et c'est surtout vrai pour Zola et Jules Romains, puisque monarchie et religion sont finalement peu envahissantes et ne structurent pas l'œuvre de Balzac comme l'hérédité les trois séries zoliennes ${ }^{13} \mathrm{ou}$ la «vie unanime» Les Hommes de bonne volonté. En outre, tout en repoussant l'hérédité, Jules Romains reprend un paradigme naturaliste, celui du corps humain, pour opposer à une construction jugée factice sa propre conception d'une «recréation organique ${ }^{14}$ ». Dès le début des dossiers préparatoires, on repérait la métaphore physiologique : «Effort pour incorporer la totalité des hommes d'une société dans cette société », c'est-à-dire celle du «premier tiers du siècle»; «B. ÉLÉMENTS À INCORPORER 15 ».

Le choix du mouvement est présent dans les injonctions que Jules Romains s'adresse à lui-même dans ses dossiers préparatoires - dont il faut préciser d'emblée qu'ils sont beaucoup moins volumineux que ceux des séries de Zola, pour un nombre de livres quasi équivalent : vingt-six romans, mais partagés en trois séries, pour Les Rougon-Macquart, Les Trois Villes et les Évangiles, vingt-sept pour Les Hommes de bonne volonté. La coïncidence aurait été parfaite si Zola avait eu le temps d'écrire Justice, son quatrième «Évangile». Le statisme est donc l'écueil à éviter :

Donner une grande importance à, ne jamais perdre de vue : Les questions de tonus, de recherche du tonus, de perception intérieure d'aise.

12. Par-delà les cas particuliers, et même s'il faut souligner que monarchie et religion sont des principes externes, alors que l'hérédité, transmise par le sang et les gènes, est un principe interne.

13. L'hérédité est en effet un principe que Zola a conservé dans les deux séries qui suivent les Rougon-Macquart : Pierre Froment, héros des Trois Villes, tient à la fois de sa mère, fervente catholique, et de son père, scientifique et athée. À leur tour, ses fils, dans les Évangiles, hériteront de la bonne hérédité transmise par Pierre et par sa femme Marie.

14. Cahiers Jules Romains 5, op. cit., p. 246.

15. Dossiers généraux, NAF 28403, fos 4 et 9; Cahiers Jules Romains 5, op. cit., p. 95 . 
En montrer le rôle, non pas statique dans les analyses d'humeur, d'états de sensibilité, mais dynamique dans la préparation des actes, l'envie d'agir, la recherche de certaines directions ou issues, l'orientation des destinées, etc. ${ }^{16}$.

On lit également, en matière d'autoconsigne :

Éviter le ton roman XIX. Cette lourdeur contenue du passé. Cette monotonie.

Beaucoup de tons. Beaucoup de procédés différents.

Mettre souvent gaîté, comique.

Toutes les techniques.

Style narratif. Monologue intérieur. Rapport. Documents directs, etc. ${ }^{17}$.

Donc, le dynamisme dans la façon de concevoir les intrigues a son corollaire dans le souci d'une variété formelle qui peut être dangereuse, mais qui était dans l'air du temps depuis la tentative de renouvellement romanesque des Faux-Monnayeurs, que Gide avait manqué en employant plusieurs procédés usés, dont l'intrusion d'auteur18. Jules Romains, voulant utiliser toutes les techniques, évitera précisément cet écueil. En revanche, il donne un titre à tous ses chapitres, comme Hugo ; c'est aussi une réminiscence du roman-feuilleton, étrangère à Zola, et que Balzac réservait aux parties de certains romans longs (Illusions perdues, Splendeurs et misères des courtisanes...).

Même si Jules Romains choisit le double garde-fou d'un vaste projet initial et de notes préparatoires, ce qui l'amène à être classé dans la catégorie des écrivains «à programme», il aime trop la liberté pour se laisser enfermer dans des plans trop stricts. C'est pourquoi les schémas préparatoires sont généraux et concis. L'écrivain s'en est expliqué dans une interview accordée à Frédéric Lefèvre en novembre 1933, alors qu'il venait de publier les tomes V et VI de sa suite romanesque :

Les plans des différents volumes des Hommes de bonne volonté ont été établis avant que je ne commence à écrire le premier tome. Mais lorsque j'en arrive à l'exécution de chaque volume, je reprends le plan qui le concerne, je le pousse un peu plus dans les détails : j'y fais des adjonctions et des modifications s'il y a lieu. En apparence, mes plans sont peu détaillés. Ils contiennent un certain nombre de points de repère dont chacun correspond dans mon esprit à une vision détaillée des personnages et des événements ${ }^{19}$.
Générales et concises, les fiches qu'il établit pour ses personnages principaux le sont tout autant. Loin de raconter toute leur histoire et de les décrire, comme Zola le fait dans ses dossiers préparatoires pour l'ensemble de ses personnages, Jules Romains reste économe de détails quand il conçoit Jallez et Jerphanion (primitivement nommé Jerfanion), chacun illustrant moins un type qu'un principe («le spirituel»; le «temporel $20 »)$. Les imaginant d'emblée ainsi, Jules Romains crée une difficulté interne car ces catégories s'assortissent mal avec le dynamisme désiré. En fait, c'est la dimension d'apprentissage des Hommes de bonne volonté qui sauvera cette contradiction interne, présente dès la conception de la série. S'y ajoutera, à partir de Recherche d'une Église (t. VII paru en 1934) une diversification encore plus grande des personnages et de l'intrigue : «Tout en restant centré sur Jerphanion, Clanricard et Laulerque, [le roman] va éclater dans de nombreuses directions 21 . » On peut reprendre à Gabriel Marcel, faisant en 1932 dans la revue L'Europe nouvelle l'éloge des Amours enfantines et d'Éros de Paris ${ }^{22}$, la notion d' «espaces libres» : ils existent en effet dès les dossiers préparatoires; alors que Zola, sans s'interdire de

16. NAF 28403, f ${ }^{\circ} 63$, ibid., p. 112.

17. Ibid., $\mathrm{f}^{\circ} 62$.

18. «Si le procédé des interventions de l'auteur ne nous choque pas chez Thackeray, Dickens ou Stendhal, c'est qu'à leur époque le roman ne cherchait pas encore à faire plus vrai que nature » (Claude-Edmonde Magny, Histoire du roman français depuis 1918, Paris, Éditions du Seuil, coll. «Points», 1971 [1950], p. 233).

19. «Une heure avec Jules Romains », interview publiée dans Les Nouvelles littéraires du 25 novembre 1933, reprise dans le Bulletin des Amis de Jules Romains, $\mathrm{n}^{\circ} 15,1979$, p. 63. Cf. Ai-je fait ce que j'ai voulu?, où Jules Romains écrit : «Mon plan s'est corrigé et concrétisé peu à peu sur plusieurs centaines de feuilles et feuillets de tailles diverses, dont je n'ai conservé qu'un petit nombre et un peu au hasard [...]. Il s'ensuit que j'ai moi-même quelque difficulté à reconstituer toutes les étapes de mon travail d'alors» (Paris, Wesmael-Charlier, 1964, p. 121). Il ne reste que cent vingt-huit feuillets, classés par l'auteur lui-même en onze sections : «grands thèmes conducteurs, éléments à incorporer, morceaux, ressorts et mouvements de l'action, technique, onomastique, personnages principaux, secondaires, relations entre les personnages, personnages collectifs, plan du tome I».

20. Ibid., fos 81-82. Jules Romains typifie aussi. Voir le calepin de 1932, $\mathrm{f}^{\circ} 27 \mathrm{v}^{\circ}$, où il présente des types d'ecclésiastiques (Cahiers Jules Romains 5, op. cit., p. 178-179).

21. Annie Angrémy, Cahiers Jules Romains 6, op. cit., p. 22.

22. T. III et IV des Hommes de bonne volonté, publiés en 1932, comme les deux premiers (Le 6 octobre et Crime de Quinette). 
changer non seulement des détails, mais aussi même des points essentiels (ainsi Étienne Lantier primitivement destiné à devenir « la bête humaine», est remplacé, pour des raisons de cohérence interne, par Jacques, un autre fils que Zola donne à Gervaise en cours d'élaboration de son roman sur le criminel, évitant ainsi d'insurmontables contradictions), conçoit ses dossiers le plus complètement possible. Si la nature a horreur du vide, le naturalisme zolien, qui est avant tout une méthode, partage cette aversion.

Dans les notes de sa conférence de 1934-1935, Jules Romains évoquait son projet en termes de «roman-maison» et de «roman-ville»; mais la comparaison n'est pas adéquate, car la maison se révèle un paradigme bien trop étroit, et la ville l'est encore, dans une moindre mesure. La construction romanesque, ici, repousse les murs et les limites spatiales. Jules Romains veut disséminer et fractionner :

La description et connaissance (positive, lyrique) de Paris se faisant à maintes reprises, par étapes, morceaux ou progrès, suivant la succession des découvertes,

ou les phases de la vie de la ville,

ou les changements et époques 23 .

Mais il souhaite aussi élargir : on passe ainsi de «Présentation de Paris à cinq heures du soir» (Le 6 octobre), à «Présentation de la France en juillet $14 »$ (dernier chapitre du Drapeau noir), puis à «Présentation de l'Europe en octobre 1933» (Le 7 octobre). Koenraad Geldof, qui a étudié la spatialisation narrative particulière des Hommes de bonne volonté, remarquait que «le récit fonctionne sur le mode d'une prolifération d'espaces concomitants, simultanés mais différents. [...] Ce mode de représentation se distingue d'autres perspectives spatiales par le fait que le mouvement et la coexistence d'espaces différenciés l'emportent sur la description véridique et photographique et avant tout sur l'idéologisation de l'espace 24 ». Les rapports à l'espace sont multipliés (K. Geldof parle de «polyperspectivisme 25 »), ce qui donne à la construction générale mobilité et souplesse. Empruntant à Michel de Certeau, dans le premier volume de L'Invention du quotidien, l'expression «récit d'espace», K. Geldof écrivait à propos de la narration des Hommes de bonne volonté : «C'est un "récit d'espace" dans lequel l'expérience dynamique plutôt que la description statique donne le ton, les pratiques de l'espace plutôt que l'intention cartographique, les gestes énonciatifs plutôt que l'inventaire des énoncés ${ }^{26}$. $\gg$ Jules Romains crée un espace romanesque pluridimensionnel où il élabore des séquences en contrepoint, ou bien les entrecroise, ou encore juxtapose les notations brèves se référant à des lieux séparés et des personnages différents, et compose un montage 27. La dilatation de l'espace romanesque a pour corollaire le décloisonnement. L'alternance, la concomitance, l'enchaînement, le passage sont des caractéristiques de cette large composition où privé et public, fiction et réel 28 abolissent aussi leurs frontières.

Pour autant, «chaque tome doit pouvoir être compris isolément 29 », note Jules Romains dans le dossier des Hommes de bonne volonté. Cette condition est en effet essentielle au maintien d'un lectorat : les lecteurs ne sont pas tenus d'attendre le vingt-septième et dernier volume pour aborder le cycle, ils peuvent également lire les volumes dans le désordre, et ne pas les lire tous, même si seule la lecture méthodique de l'ensemble donne pleine mesure de ce que l'auteur a cherché à faire : la création d'une force «en croissance», pour emprunter à Saint-John Perse, dans Vents, une expression qu'on peut appliquer à l'entreprise de Jules Romains. C'est sur ce point qu'il s'éloigne le plus nettement du Zola des RougonMacquart et des Évangiles, et cela tient au fait que dans ces deux séries - contrairement aux Trois Villes -, on ne suit pas roman après roman l'évolution du personnage principal ${ }^{30}$ et

23. Dossier préparatoire, [60] fo 802, Cahiers Jules Romains 5, op. cit., p. 111

24. Koenraad Geldof, «Le roman unanimiste ou le risque de narrer», dans Jules Romains ou les écritures de la simultanéité, dir. D. Viart, Lille, Presses universitaires du Septentrion, 1996, p. 76-77.

25. Ibid., p. 78.

26. Ibid., p. 82.

27. À propos du montage, voir Aude Leblond, Sur un monde en ruine. Esthétique du roman fleuve, Paris, Champion, 2015, p. 428-435, où elle analyse la construction du chapitre du Drapeau noir, «Tourbillon de feuilles avant l'orage».

28. Dès les premières lignes du feuillet de 1925, on lit une liste de noms de personnes vivantes à l'époque, ou quelques années auparavant, comme Duhamel (qui avait commencé l'écriture du cycle de Salavin, et allait en écrire un plus long dans les années trente, la Chronique des Pasquier), Babinski, Lapicque, Adrienne Monnier, ou encore deux assassins, Landru (qui avait été le garagiste de Jules Romains) et $\mathrm{M}^{\mathrm{me}}$ Bessarabo.

29. «Les dossiers généraux », Ms I, [59] f 800, Cahiers Jules Romains 5, op. cit., p. 111.

30. Il y en a un dans Les Trois Villes, mais deux dans Les Hommes de bonne volonté; et contrairement à Pierre Froment, présent à tout instant de Lourdes, Rome et Paris, Jallez et Jerphanion sont parfois absents de tel ou tel des vingt-sept volumes. 
d'un certain nombre d'autres, mais que chaque volume est clos sur lui-même, indépendant (alors que si chaque volume des Hommes de bonne volonté peut être lu isolément, les romans n'en découlent pas moins les uns des autres) et que la clef de voûte de l'édifice, l'hérédité, emprisonne les personnages dans un déterminisme qui n'autorise pas le dynamisme à l'échelle macrostructurale. De ce fait, la récapitulation des tares familiales faite dans Le Docteur Pascal par le héros éponyme n'apporte aucun progrès, au sens premier du terme, dans la compréhension générale de la série.

Les liens des volumes les uns avec les autres ne se manifestent pas seulement à l'échelle des vingt-sept romans. Parfois, les volumes composent des diptyques, et le directeur littéraire des éditions Flammarion Max Fischer le souhaitait dès le début, redoutant qu'un seul livre soit incapable de donner cette impression d'ensemble essentielle à la compréhension au moins partielle de ce «roman aux dimensions inusitées », porteur d'une grande ambition. Il écrivit à Jules Romains le 21 août 1931 après la lecture du premier tome :

Le 6 octobre ce sont les tout premiers tableaux d'une pièce en trente tableaux, ce sont les fondations et les caves d'un édifice. C'est un fragment. Ou c'est un soubassement. Il serait, je crois, dangereux de publier un tome comme celui-là isolément - même précédé d'un prologue. [...] Si deux tomes commencent à donner une impression d'ensemble, il y aura lieu (à mon avis) de publier deux tomes simultanément; mais si trois tomes sont nécessaires pour donner ou hâter cette impression, il faudrait se résoudre à en sortir trois à la fois 31 .

Un an plus tard, il maintint le souhait d'un tel rythme, incongru dans le monde de l'édition, mais rendu nécessaire par la nature de l'œuvre :

J'ai été content d'apprendre que votre III était terminé. Et votre IV commencé.

Vous m'avez demandé mon sentiment relativement à la façon dont il faudrait publier ce III et ce IV.

J'ai, à ce sujet, une opinion très nette : continuons à jumeler. $\mathrm{Ne}$ faisons pas d'unijambisme. Le bijambisme nous a trop bien réussi. Il y a toutes les chances pour qu'il nous soit favorable, encore, à l'avenir.

Si le sujet du IV vous le permet, essayez de donner un IV qui forme un tout, lui aussi, comme votre III $^{32}$.

Le rythme de deux volumes (qui ne forment pas toujours pour autant une paire) a été tenu presque chaque année 33 .
Parmi les diptyques, on relève Mission à Rome, treizième volume des Hommes de bonne volonté, et sa suite, Le Drapeau noir, où l'action est résolue. On peut lire les deux romans séparément, et s'arrêter au premier, qui forme un tout; mais on ne les lit pas comme Pot-Bouille et $A u$ Bonheur des Dames, ou encore La Curée et L'Argent. En effet, d'une part l'action de Mission à Rome et celle du Drapeau noir est la même, qui se poursuit d'un roman à l'autre ${ }^{34}$, alors que les romans de Zola qui viennent d'être évoqués relatent deux tranches de vie d'Octave Mouret et de Saccard; d'autre part, si l'on s'en tient à Mission à Rome, on manque le point culminant 35 , l'entrevue de l'abbé Mionnet avec le sulfureux cardinal Merry del Val, objet de l'enquête pour laquelle Mionnet est envoyé à Rome par Poincaré, et donc fréquemment nommé, mais qui n'apparaît en tant que personnage que dans Le Drapeau noir. Dans Rome de Zola aussi Pierre Froment rencontrait un homme d'Église ayant réellement existé : le pape Léon XIII ${ }^{36}$. Le rendez-vous secret donné au Vatican représentait l'acmé du roman, la fin d'une longue attente et la résolution de la venue du jeune prêtre dans la ville éternelle; dans le cas de Jules Romains, la structure est décentrée, ce qui rompt

31. Lettre citée dans les Cahiers Jules Romains 5, op. cit., partiellement p. 72, intégralement p. 270-274.

32. Lettre de Max Fischer, 13 juillet 1932, ibid., p. 274.

33. Il y eut quelques exceptions pendant la guerre.

34. Elle est toutefois plus resserrée dans Mission à Rome, plus dispersée dans Le Drapeau noir.

35. De ce roman, mais aussi de l'ensemble, comme le pensait un ancien condisciple rue d'Ulm de Jules Romains, resté son ami : «La scène entre Mionnet et Merry del Val me semble, dès maintenant, l'un des points culminants (comme elle en est, au milieu des vingt-sept tomes, le point central) de l'œuvre entière » (lettre d'André Cuisenier du 29 décembre 1937, Cahiers Jules Romains 5, op. cit., p. 60).

36. Dans Le Drapeau noir, le chapitre VII est le pendant du chapitre XIV de Rome : Mionnet y est reçu en audience privée par Merry del Val, comme Pierre Froment l'était par Léon XIII. Mais l'entrevue ne marque pas la fin du livre, parce qu'elle ne constitue pas le rêve du personnage principal. Même si Mionnet satisfait sa curiosité en ayant le privilège de rencontrer, lui simple prêtre français, l'un des plus hauts dignitaires de l'Église, et que cette rencontre représente le couronnement de sa mission, il n'y a aucune attente spirituelle : Mionnet veut protéger l'Église, mais il n'a pas les préoccupations sociales de Pierre Froment. En outre, Zola a voulu non seulement retracer mais expliquer l'histoire de la ville depuis l'Antiquité. Cette ambition est tout à fait absente du diptyque de Jules Romains. 
les habitudes de lecture et introduit un effet de surprise. Si la France laïque et républicaine envoie un de ses prêtres enquêter sur le secrétaire d'État du pape, ce n'est pas pour une simple affaire de mœurs 37 - en soi infiniment choquante pour l'Église, mais indifférente au gouvernement français; c'est parce que le jeune et brillant cardinal est viscéralement francophobe. De fait, les deux romans s'inscrivent dans un long processus de représentation de la France et d'une partie de l'Europe lors d'une période de changements profonds, de 1908 à 1933, incluant donc la montée des périls, la Première Guerre mondiale, puis une partie des troubles de l'entre-deux-guerres; et Le Drapeau noir s'achève sur une «présentation de la France en juillet 14 » qui montre le pays au seuil du conflit. Mais la série suit son cours, et s'il est important pour Mionnet de rencontrer Merry del Val, il est aussi important pour le lecteur d'avoir des nouvelles de personnages qu'il suit depuis Le 6 octobre. D'où le titre du chapitre XVII du Drapeau noir, qui n'a plus rien à voir avec les affaires vaticanes : «Qu'est donc devenu Quinette?» Quant à Jallez et Jerphanion, absents de Mission à Rome, ils resurgissent dès le premier chapitre du Drapeau noir. Comme les deux romans sont publiés séparément, l'auteur ne s'oblige à aucune transition entre l'un et l'autre. Par conséquent, Mission à Rome s'achève non sur un dénouement, mais sur un dialogue en suspens entre Mionnet et un maître-chanteur en possession d'une photographie compromettante du cardinal Merry del Val, qu'il cherche à vendre à la France - on ignore alors l'usage que feront l'abbé Mionnet et le gouvernement français de celle-ci -, et Le Drapeau noir commence in medias res sur un dialogue entre Jallez et Jerphanion qui ne concerne en rien les affaires romaines, auxquelles ils demeurent étrangers. Semblable structure narrative ne se trouve nulle part dans l'œuvre de Zola; et ces changements diégétiques entraînent une conception modifiée du temps. Siegfried Kracauer, réfléchissant à la conception herderienne du temps, a attiré l'attention sur le fait qu'

à un moment historique donné [...], nous avons affaire à une quantité d'événements qui, en raison de leur place dans des domaines différents, ne sont simultanés que dans un sens formel. En fait, la nature de chacun de ces événements ne sera correctement définie que si nous prenons en compte la position qu'il occupe dans sa séquence particulière. Les formes du temps propres à chacun des domaines éclipsent le cours uniforme du temps. [...] l'histoire est faite d'événements dont la chronologie ne nous dit pas grand-chose sur leurs rapports et sur leurs significations. Comme les événements simultanés sont le plus souvent intrinsèquement asynchrones, il est assez absurde de vouloir se représenter le processus historique comme un flux homogène. Une telle image ne fait que voiler les temporalités divergentes dans lesquelles les séquences concrètes d'événements historiques se matérialisent. S'agissant de l'histoire, on devrait parler de la marche des temps plutôt que de la «marche du temps ${ }^{38}$ ».

Dans Les Hommes de bonne volonté, l'unité chronologique se dissout au profit de carrefours spatio-temporels; se succèdent des situations marquées par la discontinuité, des cellules que le narrateur juxtapose, et qui ne donnent plus l'impression d'un écoulement temporel. La «tranche de vie» est devenue plurielle en même temps que fragmentée : au fil des volumes, Jallez, Jerphanion, Quinette, Mionnet, Gurau, Clanricard et beaucoup d'autres se croisent, s'éloignent, suivent des itinéraires parallèles, sans dépendre d'un rapport de causalité. C'est encore une différence fondamentale avec les romans réalistes et naturalistes. Il n'y a plus de nécessité guidant les personnages; pour autant, le récit ne dépend nullement de l'aimantation des mots, de cette «causalité intérieure ${ }^{39}$ » que Claude Simon défendra comme la seule possible à ses yeux : Les Hommes de bonne volonté se situent dans un entre-deux. L'enchaînement narratif est commandé par la géométrie d'ensemble; la nécessité diégétique est exclusivement conçue à grande échelle. Cette configuration particulière va de pair avec l'absence

37. Soucieux de ne pas écraser le lecteur sous son omniscience, mais de lui faire découvrir les éléments progressivement, le narrateur fait planer le mystère sur le caractère «délicat» de l'affaire qui conduit le ministre Gurau, personnage fictif, à demander à Raymond Poincaré la permission de diligenter une enquête secrète sur Merry del Val. Les mœurs de celuici étaient bien connues, et la photo dont il est question a existé, selon Jules Romains (voir Cahiers Jules Romains 6, op. cit., p. 346. Jules Romains écrit cette longue lettre à un destinataire non identifié, le 26 mai 1953. Il trouvait absurde le projet de béatification de Merry del Val, lancé le 26 février 1953, et qui n'aboutit pas).

38. Siegfried Kracauer, L'Histoire. Des avant-dernières choses (History. The Last Things Before The Last, New York, Oxford University Press, 1969), trad. Cl. Orsoni, préface de Jacques Revel, Paris, Stock, 2006, p. 214-216.

39. Claude Simon, Discours de Stockholm (10 décembre 1985), Paris, Éditions de Minuit, 1986, p. 22. 
de transition, difficilement concevable dans un roman du XIX ${ }^{\mathrm{e}}$ siècle, même si Bouvard et Pécuchet ouvrait des voies nouvelles en ce domaine ${ }^{40}$.

Bien que diplômé en sciences aussi bien qu'en philosophie, et souhaitant laisser également son nom dans ce domaine (ce qui ne fut pas le cas, et l'échec de la réception de ses découvertes sur la rétine fut une vive déception), Jules Romains ne s'est pas appuyé comme Zola sur le paradigme scientifique pour écrire ses romans. Il n'en a pas moins conçu, avec ses Hommes de bonne volonté, un roman expérimental, invitant à bouleverser les habitudes de lecture d'une série ou d'un cycle. En effet, on l'a vu, chaque roman peut se lire séparément, mais ce n'est qu'en lisant la totalité qu'on prend conscience de ce que l'auteur a voulu réaliser, et qui n'a pas d'équivalent dans la littérature française. Pour autant, est-il parvenu à faire ce qu'il voulait 41 ? Il aurait pu juxtaposer des possibles contradictoires, expérimenter d'autres schémas narratifs, diminuer la part des dialogues et travailler sur les mots et les images plus que sur les idées; mais le fond du roman du XIX ${ }^{\mathrm{e}}$ siècle resurgit, alors même qu'il voulait le fuir ${ }^{42}$. Jules Romains fragmente, mais n'en poursuit pas moins une continuité, et, à travers les chaos de l'Histoire comme de ses personnages, il suit malgré tout un fil chronologique et construit une totalité cohérente. Le début des dossiers préparatoires ne laisse aucun doute sur ce point. L'écrivain ne nie pas le désordre, mais tente de le résorber dans le sens à donner au fil des volumes :

Faire comprendre (au besoin en le disant expressément, à certains moments) comment une époque peut être à la fois incohérente allant n'importe où, et, quant à l'ensemble et avec recul, orientée.

D'un des tomes à l'autre, accentuer le sentiment de cette orientation 43 .

De fait, la réception de son œuvre, dès le début, témoigne d'une concentration et non d'une dispersion : «On s'habitue très bien à cette simultanéité d'actions et de peintures diverses, et une impression d'énorme unité se dégage de cette lecture», lui écrit son ami Henri Legrand ${ }^{44}$. Or, unité et cohérence sont bien des impératifs de la création réaliste et naturaliste; et même si l'unanimisme, au principe des Hommes de bonne volonté, n'est pas une structure déterministe comme l'hérédité, il reste une structure préexistant à la création, et en utilisant un principe aussi artificiel et subjectif que ceux qu'il rejetait, Jules Romains pense encore en homme du XIX ${ }^{\mathrm{e}}$ siècle. Koenraad Geldoff a attiré l'attention sur les contradictions de la préface, où Jules Romains appelle le lecteur à se défaire de ses habitudes, tout en le rassurant sur le caractère «momentané» de la désorientation ressentie. Le critique remarque que «la narration ouverte finira par se plier à une téléologie normative qui, d'invisible dans les premiers volumes, deviendra de plus en plus nette par la suite 45 ». De fait, la promesse d' «une narration autre» est sacrifiée. Cette promesse avait été permise par le passage de l'unanimisme, né en poésie, à la prose, moyen plus vaste, plus souple, donc particulièrement adapté, et générant une infinité de «possibles» narratifs exploités par d'autres écrivains. Comparant les romans de Jules Romains et de John Dos Passos, Jean-Pierre Morel, après avoir remarqué que «le seul véritable point commun aux deux romanciers est l'usage qu'ils font du récit multilinéaire et (forcément) discontinu qui permet de mener de front - en les plaçant successivement, et plusieurs fois de suite, sur le devant de la scène - plusieurs intrigues ou lignes d'action différentes, individuelles ou collectives, parallèles dans le temps de

40. Voir à ce sujet la thèse de Florence Pellegrini (dir. J. Neefs) soutenue en 2005 à l'université Paris VIII : «Mais pourquoi m'a-t-elle fait ça! » : la causalité dans Bouvard et Pécuchet de Gustave Flaubert. On lit au début de son résumé : «La préoccupation de la jonction est, chez Flaubert, obsessionnelle : la correspondance évoque à de nombreuses reprises la difficulté que constitue pour l'écrivain l'élaboration des transitions. » Toutefois, l'étude du dernier roman révèle « une dynamique qui aboutit à un amuïssement de la causalité, et cela quel que soit le domaine expérimental abordé». Elle étudie «"l'alliance anormale" entre la surabondance des marqueurs de jonction et l'effet de dislocation » («résumés de thèses », mis en ligne le 19 janvier 2009 : < http://flaubert.revues.org/591 >).

41. Cette question fait écho au titre d'un de ses livres : Ai-je fait ce que j'ai voulu?

42. Philippe Chardin, dans «De deux simultanéismes discordants : Musil et Jules Romains » (où il explique que si Musil était fasciné par la simultanéité narrative, il n'était pas attiré par l'unanimisme), montre que ces deux romanciers ont en commun le fait d'être allés beaucoup moins loin que Dos Passos dans la simplification des personnages, les associations mentales, les raccourcis, et maintiennent en outre «le point de vue d'en haut» pour «dominer le chaos qui les entoure» (Jules Romains ou les écritures de la simultanéité, op. cit., p. 214-215).

43. Dossiers généraux, [6] 5, Cahiers Jules Romains 5, op. cit., p. 96-97. 44. Lettre du 31 décembre 1932, Cahiers Jules Romains 5, op. cit., p. 284. 45. Art. cité, p. 86-87. Cf. ibid., p. 98. 
l'action et plus ou moins séparées l'une de l'autre dans l'espace », souligne que, contrairement à son confrère américain, le romancier français conserve la trame d' « un récit de facture assez traditionnelle, où la situation narrative dominante est (à l'exception des carnets de Stephen Bartlett) celle de la troisième personne, et où la narration est exercée de façon souveraine au niveau de la régie et du commentaire»; il souligne aussi «le rôle presque permanent d'exposition, de liaison et d'explication confié au narrateur

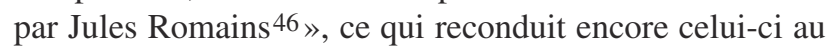
roman réaliste-naturaliste dont il voulait s'éloigner.

Le caractère épars, fragmentaire, disparate des brouillons, l'absence de structure centrale reliant entre eux les cercles du plan unanimiste, l'absence de constitution méthodique du dossier préparatoire, autant de vraies différences avec Zola, qui pouvaient laisser supposer que le romancier se déprendrait d'une totalité, et briserait les liens avec les grands exemples du XIX ${ }^{\mathrm{e}}$ siècle. Si l'on revient à ce plan de 1932 , on est confronté à une énigme, alors que les deux états de l'arbre généalogique de Zola sont lisibles par tous, y compris par ceux qui n'ont jamais ouvert ses romans. On déduit de l'ensemble que le personnage n'est plus central dans l'action, et que l'originalité de Jules Romains porte en partie sur ce point : ce sont les interactions qui prévalent. Mais les certitudes s'arrêtent là. Les vingt grappes de monades sont hétérogènes : elles vont, sans nécessité explicite, par deux, trois, quatre, cinq, six, sept, huit, dix; la plus grosse en compte seize, alors que certaines bulles restent isolées (dix au total). Le nombre additionné de grappes et de bulles (trente) ne correspond pas à celui des romans. L'agrégation est le mode de liaison dominant, mais dans une grappe centrale, la bulle «Mionnet et son milieu », agrégée à «Deux catholiques laïques fervents : l'artiste, et le savant 940, 350» est reliée par un trait à celles qui en découlent : «Le carnet de Mionnet», et «Le carnet du médecin pour nerveux»; de même pour une petite grappe figurant au bas de la page droite du plan. On s'étonne aussi de certaines absences de liaison : la bulle dans laquelle est inscrite «tableau de la France» est isolée, proche mais non collée à celle qui concerne le «tableau de l'Europe», alors même que, dans la réalité du roman achevé, France et Europe ne se dissocient pas. L'aspect matériel de ce plan, et des notes préparatoires, fait espérer une structure en tout point différente des séries du passé. On attendrait une atomisation non résolue, en accord avec les aléas de chaque existence, quand c'est finalement une superstructure qui est conçue, porteuse d'une nécessité interne qui faisait selon Jules Romains défaut aux Rougon-Macquart.

La construction des Hommes de bonne volonté obéit à deux impératifs : "Concilier la vision ubiquitaire et omnisciente avec la nécessité de découverte graduelle pour ménager l'intérêt ${ }^{47}$. » Jules Romains poursuit ainsi un but identique à celui du romancier traditionnel : il doit susciter et maintenir l'intérêt des lecteurs - rien, encore une fois, qui relève du principe interne de «recréation organique» destiné à démontrer la fausseté du «système Zola». Les découvertes progressives n'ont donc pas chez lui le même objectif que le souci de fidélité au réel qui conduisait Proust à proposer des vérités partielles corrigées ou complétées plus tard, au fil de la lucidité progressivement acquise par le narrateur. Tributaire des lecteurs, auxquels il s'adresse dans la préface sous forme de captatio benevolentiae, Jules Romains romancier fuit la poésie à laquelle il s'était consacré dans ses jeunes années, et celle-ci ne reparaît pas dans sa prose : il n'a jamais tissé dans sa longue suite romanesque de réseaux métaphoriques, qui auraient pourtant permis de «retrouver les choses par le dedans », comme l'avait fait Zola. En dépit d'une conception renouvelée de la structure d'ensemble d'une suite narrative, son vaste roman, cherchant à imiter la structure du réel, ne réussit pas à dépasser l'artificiel pour retrouver le «naturel». Claude-Edmonde Magny, avec sa lucidité coutumière, l'avait remarqué, qui définissait l'architecture des Hommes de bonne volonté comme «savante et concertée». Considérant que les différentes intrigues demeuraient «relativement compartimentées », elle voyait l'univers de Jules Romains «morcelé par l'analyse, décomposé par l'intelligence au point que la puissance créatrice ne parv[e]nait pas à en reconstituer un tout organique, doué de respiration et de vie ${ }^{48} \gg$.

46. Jean-Pierre Morel, «Jules Romains et Dos Passos. Remarques », ibid., p. $224-225$ et 234

47. Dossier, [59], f ${ }^{\circ}$ 801, Cahiers Jules Romains 5, op. cit., p. 111

48. Claude-Edmonde Magny, op. cit., p. 282. 
SOPHIE GUERMÈS est professeur à l'université de Brest et membre élue du conseil de laboratoire de l'ITEM (CNRS-ENS). Elle a publié plusieurs ouvrages, dont, pour ceux qui concernent le roman, L'Écho du dedans (Klincksieck, 1997), La Religion de Zola (Champion, 2003; Champion-classiques Essais, 2006), et La Fable documentaire (Champion, 2016).

Sophie.Guermes@univ-brest.fr

\section{«Retrouver les choses par le dedans"}

Jules Romains refuse les systèmes, auxquels il oppose un « travail organique ». Il reproche à Zola d'avoir conçu pour Les RougonMacquart une unité de façade, l'hérédité devenant à ses yeux un principe aussi artificiel qu'abstrait. Il veut aussi éviter le plus possible les pièges du sériel par la traduction littéraire de l'unanimisme et de la simultanéité. Le caractère épars, fragmentaire, disparate des brouillons, l'absence de structure centrale reliant entre eux les cercles du plan unanimiste, l'absence de constitution méthodique du dossier préparatoire sont autant de différences avec Zola ; pour autant, l'ensemble des volumes des Hommes de bonne volonté montre que Jules Romains, soucieux de ménager l'intérêt des lecteurs, et choisissant pour cette raison de composer une totalité cohérente, n'a pas réussi à briser les liens avec les grands exemples du XIXe siècle.

Jules Romains rejected systems, to which he opposed an "organic work". He reproached Zola for having conceived merely a façade of unity in Les Rougon-Macquart: heredity, that according to him became a principle as artificial as it was abstract. He wanted to avoid as much as possible the traps of the serial by opting for the literary transposition of unanimity and simultaneity. The scattered, fragmentary and heterogeneous character of his drafts, the absence of a central structure linking the circles of the Unanimist outline, the absence of a systematic constitution of a preparatory dossier, are all so many differences with Zola's method. Yet, the sequence of volumes of Les Hommes de bonne volonté shows that Jules Romains, determined to hold the readers' interest, and thus choosing to compose a coherent totality, was not able to free himself from the great 19th-century models.

Jules Romains lehnt Systeme ab und stellt ihnen das Konzept einer „organischen Arbeit“ entgegen. Er wirft Zola vor, in Les Rougon-Macquart eine nur fassadenhafte Einheit geschaffen zu haben, wobei die Vererbungstheorie in seinen Augen ein künstliches und abstraktes Prinzip ist. Er möchte auch, wenn irgend möglich, die Fallen des Seriellen meiden, indem er eine literarische Entsprechung zu Einstimmigkeit und Gleichzeitigkeit sucht. Der zerstreute, disparate und uneinheitliche Charakter der Entwürfe, das Fehlen einer zentralen Struktur, welche die verschiedenen Kreise des einstimmigen Plans miteinander verbindet, sowie das Fehlen eines methodisch angelegten „dossier génétique“ - dies alles sind Unterschiede zu Zola; trotzdem zeigen die Bände von Les Hommes de bonne volonté insgesamt, dass Jules Romains, darauf bedacht, das Interesse der Leser zu wecken, und daher bestrebt, ein kohärentes Ganzes zu schaffen, es nicht vermochte, mit den großen Vorbildern des 19. Jahrhunderts zu brechen.
Jules Romains rechaza los sistemas, a los que le contrapone un "trabajo orgánico". Le reprocha a Zola el haber concebido en Los Rougon-Macquart una unidad superficial: la herencia se convierte, en su perspectiva, en un principio tan artificial como abstracto. Trata también, en lo posible, de evitar las trampas de la serie a través de la traducción literaria del unanimismo y la simultaneidad. El carácter disperso, fragmentario, dispar de los borradores, la ausencia de una estructura central que vincule los círculos del plan unanimista entre ellos, la inexistencia de un dossier preparatorio constituido metódicamente, constituyen verdaderas diferencias con Zola; no obstante, el conjunto de los volúmenes de Hommes de bonne volonté pone de manifiesto que Jules Romains, en su intento de componer una totalidad coherente para preservar el interés de sus lectores, no ha logrado romper los vínculos con los grandes ejemplos del siglo XIX.

Jules Romains recusa os sistemas, a que contrapõe o «trabalho orgânico». Critica Zola por ter projetado em Les Rougon-Macquart uma unidade de fachada, pois a hereditariedade torna-se aos seus olhos um princípio tão artificial quanto abstrato. Igualmente pretende evitar as armadilhas do modelo serial, que encontram tradução literária no unanimismo e na simultaneidade. O caráter disperso, fragmentário e desigual dos seus rascunhos, a ausência de uma estrutura central ligando entre si os círculos do plano unanimista, a falta de um dossier preparatório constituído metodicamente são algumas das diferenças com Zola; não obstante, o conjunto dos volumes dos Hommes de bonne volonté mostra que Jules Romains, preocupado com o interesse dos leitores e com a totalidade coerente que lhes deseja proporcionar, não foi capaz de romper com os grandes exemplos do século XIX.

Jules Romains rifiuta i sistemi, ai quali contrappone un "lavoro organico". Rimprovera Zola per aver concepito nei RougonMacquart un'unità di facciata, poiché l'eredità diventa, ai suoi occhi, un principio tanto artificiale quanto astratto. Egli vuole anche evitare il più possibile le insidie della serialità, grazie alla traduzione letteraria dell'unanimismo e della simultaneità. Il carattere sparso, frammentario, eterogeneo dei brogliacci, l'assenza di struttura centrale per collegare tra loro i circoli del piano "unanimista", l'assenza di costituzione metodica del dossier preparatorio, sono altrettante differenze tra Romains e Zola; ciononostante, l'insieme dei volumi degli Hommes de bonne volonté, dimostra che Jules Romains, preoccupato di venire incontro all'interesse dei lettori, e cercando perciò di comporre una totalità coerente, non è riuscito a rompere i legami con $\mathrm{i}$ grandi esempi del XIX secolo. 